\title{
Modified LEACH-DT Algorithm with Hierarchical Extension for Wireless Sensor Networks
}

\author{
Vrinda Gupta \\ Department of Electronics and Communication Engineering National Institute of Technology Kurukshetra, 136119, \\ Haryana, India \\ E-mail: vrindag16@gmail.com \\ Rajoo Pandey \\ Department of Electronics and Communication Engineering National Institute of Technology Kurukshetra, 136119, \\ Haryana, India \\ E-mail: rajoo_pandey@nitkkr.ac.in
}

\begin{abstract}
The selection of cluster heads is of immense importance in the cluster based protocols which are widely used in wireless sensor networks. However, for the random selection of cluster heads when the distance of cluster heads is also taken into account such as in LEACH-DT algorithm, the lifetime of the network is improved. In this paper, a new algorithm for selection of cluster heads is proposed where the residual energy of the cluster heads is also considered along with the distance to the base station. The proposed approach ensures better energy balance of the network nodes. The performance of the proposed algorithm is measured at various stages of network lifetime and a significant improvement over LEACH-DT has been observed. Also, a two-level heterogeneous hierarchical architecture of the proposed algorithm is investigated which further enhances the performance of the proposed algorithm.
\end{abstract}

Index Terms - Energy consumption, Hierarchical architecture, LEACH-DT protocol, Network lifetime, Wireless Sensor Network.

\section{INTRODUCTION}

Wireless sensor network (WSN) has a wide range of applications in several areas of engineering and technology e.g. harsh physical environmental monitoring, military surveillance, smart offices, and many more. These networks comprise of sensors as nodes which are small in size and generally equipped with nonrechargeable battery. The sensors are deployed at different locations for carrying out the important tasks such as sensing, processing, and finally communicating from the field of interest to the sink which is usually placed at a far away location from the sensor field [1-2].

One of the major applications of a sensor network is remote environmental monitoring, in which an end user can monitor a distant environment by accessing the data being relayed at the sink. For this application, usually the sensor nodes are deployed in a randomized manner, i.e. they are scattered into an environment randomly. Here, these sensor nodes must co-operate to learn about their locations, know their neighbors and find paths to transmit their sensed information to the sink. These sensors are distributed in large numbers in the region to be monitored because they are prone to failures mainly because of battery exhaustion. In order to manage such a complex topology, clustering has been found to be efficient [3-4].

In the energy model used for sensor networks, communication is considered as the most energy expensive function. Thus, reducing the energy consumption in sensors communications is the main design objective in any wireless sensor networks design. Out of the various protocols developed in the past for sensor network communications, hierarchical cluster based protocol is the standard mechanism to achieve this design objective in an efficient manner [2]. In hierarchical clustering protocol, nodes have different roles to play. However, few nodes are elected as leader nodes which apart from sensing data also act as forwarding nodes to relay other nodes' data. The other nodes only transmit their own sensed data to the leader nodes.

Among the proposed cluster-based protocols for WSNs, Low Energy Adaptive Clustering Hierarchy (LEACH) approach [3], [5], [6] is perhaps the most popular and effective one. A number of variants of LEACH protocol such as an energy balancing based LEACH improvement (LEACH-B) [7], LEACHCentralized (LEACH-C) [6],[8], a distributed clustering algorithm for WSNs (LEACH-D) [9], Threshold based LEACH (T-LEACH) [10], Variable-Round LEACH (VRLEACH) [11], N-LEACH [12], Hybrid-LEACH [13], LEACH improvement with adaptive cluster-head selection and two-hop transmission (ACHTH-LEACH) [14],V-LEACH [15], LEACH-Z [16] and many more have been proposed by researchers in the past.

Some other energy efficient cluster based protocols proposed are Distributed energy efficient and Fault 
Tolerant Routing algorithm (DFTR) [17], Deterministic Cluster Head Selection (DCHS) [18], Load Balanced Clustering (LBC) [19], HEED [20], Energy Efficient Clustering Scheme (EECS) [21], Layered Clustering Hierarchy (LCH) [22], Energy-Efficient Heterogeneous Clustering scheme (EEHC) [23], CEC [24], EnergyEfficient Load Balanced Clustering Algorithm (EELBCA) [25], Density based Energy-efficient Clustering Algorithm (DECA) [26]. In these protocols, nodes are organized into a number of clusters. Each cluster has a self-elected leader node, known as cluster head $(\mathrm{CH})$. This $\mathrm{CH}$ does the long distance transmission to the sink after aggregating the sensed data received from its member nodes. Each non $\mathrm{CH}$ node is associated with its nearest cluster head and communicates with the sink only through its head node.

There are number of metrics for evaluating the performance of the sensor network, but the most important is energy efficiency and network lifetime. A network would be energy efficient if its nodes employ energy efficient algorithms in performing all its functions. A sensor node has three main functions to perform, i.e. sensing, local processing and communicating wirelessly. In a battlefield, wherein sensors are deployed to monitor a chemical activity, the number of sensors being alive is important. The lifetime of a sensor network has most commonly been defined by the term system lifetime which can be defined as the time elapsed until the first node in the network depletes its energy [27]. But using this definition of lifetime may not be appropriate as the failure of one sensor will not affect much as there is redundancy of deployed sensors. Thus, there are many definitions of network lifetime depending on the application for which the sensors are deployed [27]. So, another one could be time elapsed till certain percentage of sensors fail. We have in this paper, therefore, evaluated the network lifetime with respect to time until not only $1 \%$ sensors fail but also till $25 \%, 50 \%, 75 \%$ and $90 \%$ sensors fail. In any case, maximum lifetime will be obtained when every sensor node dissipates its energy at the same rate. Therefore, in order to achieve this goal of maximizing network lifetime, every node is important and also its energy consumption too.

In the LEACH protocol, the energy consumption among sensor nodes is asymmetric. The cluster heads which are at large distance from the sink consume their energy earlier than those near the sink thereby some nodes expire sooner than others. As a result, the network size continuously decreases and the network gets partitioned due to dead nodes and the system performance declines. Furthermore, LEACH protocol has another major drawback i.e. it assumes that all sensors can communicate with the base station (BS) or sink directly. This limits the scalability of LEACH protocol [5-6]. However, this can be easily catered by implementing a hierarchical fusion architecture, where some $\mathrm{CHs}$ have the task of gathering data from other CHs [5-6].

Few distance-based cluster head selection algorithms have been reported in literature. In [28], a distance-based segmentation scheme has been proposed wherein the difference of remaining energy of close by and distant nodes is taken care of by using different clustering policies in each segment of the network in order to prolong the network lifespan. In [29], a distance based threshold, LEACH-DT is proposed in which $\mathrm{CH}$ selection is based on assigning different probabilities with respect to the nodes distance to the sink such that the energy balance is achieved. The authors in [30] have proposed the hierarchical extensions of the LEACH [5] and LEACH-DT [29] protocols and made them suitable for large-scale networks.

In a cluster based organization, cluster head node consumes its energy in two processes. One is intra-cluster and other is inter-cluster process. One of the major causes of the problem of unbalanced energy consumption is because the clusters formed are not of even sizes. To take into account this energy imbalance, for selection of $\mathrm{CH}$, a new protocol considering the distance parameter as used in LEACH-DT as well as the residual energy parameter has been proposed in this paper. Further, a new topology, a heterogeneous hierarchical architecture of the sensor network with two tiers based on the LEACH protocol architecture at the lower level and the LEACH-DT protocol architecture at the upper level is also suggested.

The remaining paper is organized as follows. The basic LEACH protocol architecture along with another cluster head selection method namely, LEACH-DT where distance based threshold is used for $\mathrm{CH}$ selection and the energy consumption of sensor nodes in clustering approach are discussed in Section II. The proposed protocol which uses new energy-aware cluster-head selection policy is described in Section III. The heterogeneous hierarchical fusion architecture utilized for designing the hierarchical extension of the proposed protocol is presented in Section IV. The simulation and the results obtained are discussed in Section V. The paper is finally concluded in Section VI.

\section{Cluster Head Selection Algorithms}

For cluster head selection procedure, Low-Energy Adaptive Clustering Hierarchy (LEACH) protocol [5], available in literature is being widely used. It provides energy-efficient cluster based architecture and application-specific data aggregation approach.

The protocol operation is done in two steps. The first step is set-up phase. In this phase, $\mathrm{CHs}$ are chosen along with the selection of their members, leading to formation of clusters and also TDMA schedules in each cluster. The second step is steady-state phase. In the steady-state phase, the data signals are transmitted from members to $\mathrm{CH}$ and from $\mathrm{CH}$ to $\mathrm{BS}$. $\mathrm{LEACH}$ rotates the role of $\mathrm{CH}$ among sensor nodes in order to evenly distribute the energy load as being a $\mathrm{CH}$ consumes lot of energy.

The protocol operation is done in rounds, where a round refers to operation of above two steps leading to data communication from the sensing field to the BS. At the beginning of each round, every sensor node elects it- 
self to be $\mathrm{CH}$ with a certain probability. The desired percentage of $\mathrm{CHs}$ is given by $\mathrm{p}=\mathrm{k} / \mathrm{N}$ in each round, where $\mathrm{N}$ is the number of sensor nodes distributed in a field and $\mathrm{k}$ is the expected number of $\mathrm{CHs}$ [6].

In LEACH protocol, 1/p successive rounds are combined together known as group of rounds (GOR). A node gets itself elected in a round with probability $\left(\mathrm{T}_{\mathrm{i}, \mathrm{r}}\right)$ [6], [30] given as follows:

$$
T_{i, r}=\left\{\begin{array}{cl}
\frac{p}{1-p\left(r \bmod \left(\frac{1}{p}\right)\right)}, & i \in G(r) \\
0, & \text { otherwise }
\end{array}\right.
$$

where $G(r)$ is the group of nodes which have not acted as $\mathrm{CHs}$ in that group of round.

For achieving better balancing of the energy consumption, a distributed cluster head selection method, LEACH-DT is proposed which is based on distance based threshold [30]. In this protocol, each node is assigned a different probability based on how far it is away from BS. Here, instead of $p$, a fixed pre-defined system parameter of LEACH, a differentiated percentage $\mathrm{p}_{\mathrm{i}}$ of $\mathrm{CHs}$ as a function of the distance to the sink is used. $\mathrm{p}_{\mathrm{i}}$ is computed as shown in equation (2).

$$
p_{i}=\frac{k * \varepsilon_{i}}{\sum_{j=1}^{N} \varepsilon_{j}},\left(0 \leq p_{i} \leq 1\right)
$$

where $\varepsilon_{\mathrm{i}}=1 /\left(\mathrm{E}_{\mathrm{CH}}\left(\mathrm{d}_{\mathrm{i}}\right)-\mathrm{E}_{\text {non-CH}}\right)$. Here $\mathrm{E}_{\mathrm{CH}(\mathrm{di})}$ and $\mathrm{E}_{\text {non- } \mathrm{CH}}$ are the average amount of energy consumption in a round by $\mathrm{CH}$ node and non- $\mathrm{CH}$ node described in the next subsection $\mathrm{A}$. In round $\mathrm{r}$, a node i elects it-self to become a $\mathrm{CH}$ with probability $\mathrm{T}_{\mathrm{i}, \mathrm{r}}$ [30] defined as below.

$$
T_{i, r}=\left\{\begin{array}{cc}
\frac{p_{i}}{1-p_{i}\left(r \bmod \left(\frac{1}{p_{i}}\right)\right)}, & G_{i}(r)=0 \\
0 & G_{i}(r)=1
\end{array}\right.
$$

\section{A. Energy Consumption Analysis}

In this part, the energy dissipation of a cluster head node and a non cluster head node in a round are analyzed. It is assumed that all the messages except the ADV message are transmitted through spreading code system with spreading factor, $h$ [30]. The energy dissipation of a cluster head node in a round is according to equation (4).

$$
\begin{aligned}
& E_{\mathrm{CH}(d i)}=E_{\mathrm{mac}}+E_{T}\left(l_{\mathrm{adv}}, \sqrt{ } 2 M\right)+\operatorname{count}(i) * E_{R}\left(h, l_{\mathrm{join}}\right) \\
& \quad+E_{T}\left(h * l_{\mathrm{TDMA},} \sqrt{2 M}\right)+\operatorname{count}(i) * E_{R}\left(h * l_{\mathrm{data}}\right) \\
& \quad+E_{\mathrm{DA}}(\operatorname{count}(i)+1)\left(l_{\mathrm{data}}\right)+E_{T}\left(h * l_{\text {data }}, d_{i}\right)
\end{aligned}
$$

where $\mathrm{E}_{\mathrm{CH}(\mathrm{di})}$ is the average amount of energy consumption of a $\mathrm{CH}$ node which is at a distance $\mathrm{d}$ from the BS. $\mathrm{E}_{\mathrm{mac}}$ is the energy expense for CSMA used for communication between $\mathrm{CHs}$ and the base station. $\mathrm{E}_{\mathrm{T}}$ $\left(\mathrm{l}_{\mathrm{adv}}, \sqrt{2} \mathrm{M}\right)$ corresponds to the energy expense for $\mathrm{CH}$ node while it broadcasts an advertisement (ADV) message of length $1_{\text {adv. }}$ Each non- $\mathrm{CH}$ node chooses a nearest $\mathrm{CH}$ node and transmits a join-request (JOINCLUSTER) message to the chosen $\mathrm{CH}$. $\mathrm{CH}$ then simultaneously broadcast TDMA scheduling (TDMASCHEDULE) message corresponding to distance $\sqrt{ } 2 \mathrm{M}$ meters for data transmission. $\operatorname{count}(\mathrm{i}) * \mathrm{E}_{\mathrm{R}}\left(\mathrm{h} * \mathrm{~h}_{\text {data }}\right)$ corresponds to the energy expense by a $\mathrm{CH}$ node in receiving the sensed information (data signals) from its cluster members being represented by count(i). $\mathrm{E}_{\mathrm{DA} * \operatorname{count}(\mathrm{i}) * l_{\text {data }}}$ refers to the energy expense in the data aggregation process. A $\mathrm{CH}$ node finally transmits $1_{\text {data }}$ to BS which is at a distance $d_{i}$. The energy dissipation of a non- $\mathrm{CH}$ node in a round is given by equation (5).

$$
\begin{gathered}
E_{\mathrm{nonCH}}=k * l_{\mathrm{adv}} * E_{R}+E_{T}\left(h_{*} l_{\text {join }}, \sqrt{ } 2 M\right)+E_{R}\left(h * l_{\mathrm{TDMA}}\right) \\
+E_{T}\left(h * l_{\text {data }}, \text { dis }\right)
\end{gathered}
$$

Here $E_{\text {non-CH }}$ is the average amount of energy consumption in a round by a non- $\mathrm{CH}$ node and dis is the distance of the non- $\mathrm{CH}$ node from its $\mathrm{CH}$ node.

\section{PROPOSED APPROACH}

In order to achieve maximum network lifetime in WSN, energy consumption must be well balanced among nodes. To achieve this goal, every node in the network is given equal probability for becoming $\mathrm{CH}$ in $\mathrm{LEACH}$ protocol [5-6]. But the clusters formed are of uneven sizes. As seen in energy consumption analysis, the $\mathrm{CH}$ consumes energy proportional to number of cluster members it has in receiving JOIN messages and data signal messages, further in aggregating the data signal messages. In this intra-cluster processing, the problem of energy imbalance is created. The proposed protocol focuses on this energy imbalance problem. After completion of one GOR execution, some $\mathrm{CHs}$ may drain out their residual energy more than others. So, in order to balance the load, after first GOR execution, this protocol considers both distance and energy factors while selecting cluster heads. The protocol is designed as follows.

Assume an $M$ x $M$ square sensor field in which $N$ nodes are distributed randomly. The sensor field is divided into fixed number of equal area segments such that the nodes in each segment are almost at the same distance to the base station. A node selects itself as $\mathrm{CH}$ at the beginning of round $r$ with probability $p_{i}$ and compares the chosen random number with threshold obtained from equation (3). If the number chosen is less than a threshold $T_{i, r}$, the node is elected as cluster-head node for that round. The protocol works as LEACH-DT in the first GOR i.e. until each node of every segment has executed the $\mathrm{CH}$ role once. After this first GOR execution, in order to achieve load balance, the cluster-head after being selected through random selection process of LEACHDT, transfers its role to another eligible node in the same segment in which it is located, one which has maximum residual energy $\left(E_{\text {current }}\left(s_{m}\right)\right.$ at that particular time. Thus, the old cluster-head which was selected in a particular round $r$ gets replaced by a new $\mathrm{CH}$ node lying in the same segment as that of old $\mathrm{CH}$ and having highest residual energy.

The pseudo-code for constructing new cluster-head 
selection is given in Algorithm 1. In each of the segment where old cluster head was self-elected, a new cluster head is selected through this algorithm.

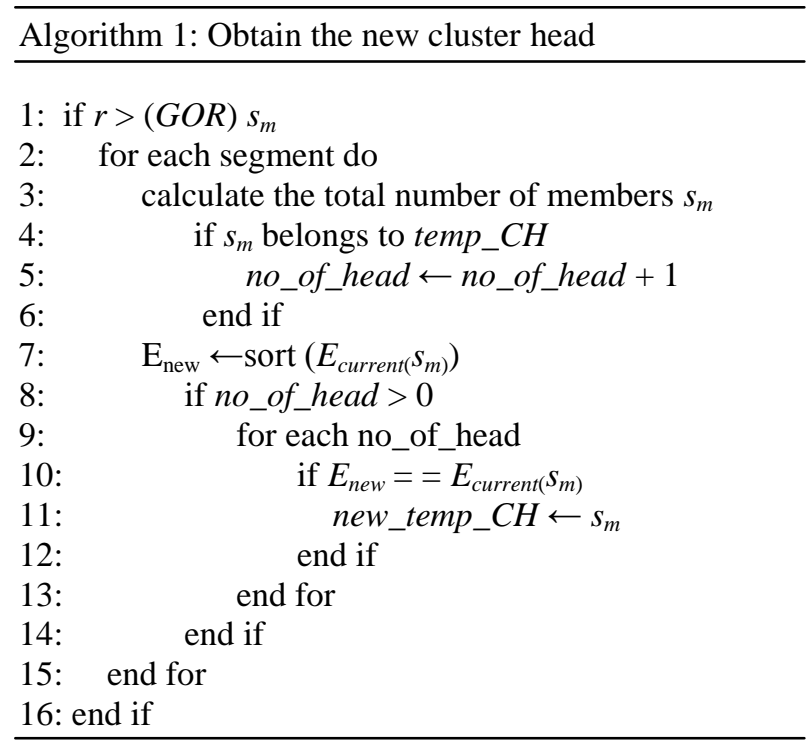

After $\mathrm{CH}$ election sub-phase, then in next sub-phase, each non-cluster head node associates with one of the selected cluster heads for that round. This cluster head selection is through the advertisement that the finally selected cluster heads broadcast indicating that they have become cluster heads. The advertisement message contains the node ID. All the sensor nodes receive these advertisements. If a node hears advertisements from a number of cluster heads, then the head to which it gets associated is based on the signal strength of the received ADV messages. The message received with the highest signal strength is selected as their cluster head. The sensor nodes inform about their association to the cluster heads in the cluster set-up sub-phase. Based on the number of member nodes in the cluster, the cluster-head node prepares a communication schedule and receives the data signals in the steady-state phase. The clusterhead node performs aggregation function on the received data signals and compresses all the received signals into a single common signal. This composite data is then transmitted to the base station. After completion of one round of communication another round of operation begins. The network enters into first step i.e. set-up phase again and selects the cluster heads through the same algorithm.

\section{Hierarchical Extension OF Proposed Protocol}

In large scale networks, where distance between some sensors and the BS is large, single-hop LEACH-DT is not suitable due to power limitation of a sensor. In order to address this problem, heterogeneous hierarchical fusion architecture is applied. The lower level of clusterheads is selected using LEACH protocol whose task is to transmit data signal to their respective super cluster head. The upper level cluster-heads, Super cluster heads $\left(S_{C H}\right)$, is selected using LEACH-DT protocol which have to transmit the data signals to the BS.

The algorithm is to be repeated in every communication round. In every round several $\mathrm{CHs}$ are elected using randomized procedure with same predefined Threshold, $T$ defined in equation (1). Then super cluster heads election is carried out from the eligible nodes who are $\mathrm{CH}$ in that particular round i.e. temp_CH nodes again go through election procedure for $S_{C H}$ election with $p_{i}$ given through LEACH-DT protocol. If a super cluster head node $S_{C H}$ has been selected in a segment, then the current energy level i.e. the residual energy of every member nodes (CHs) of that segment is checked. If the temporary selected $S_{C H}$ node's remaining energy is maximum then it remains as new $S_{C H}$, otherwise it is replaced by a new $S_{C H}$, the one whose residual energy is highest. Thus node having the highest energy value is finally selected as new $S_{C H}$. The new super cluster head selection algorithm is illustrated in Algorithm 2.

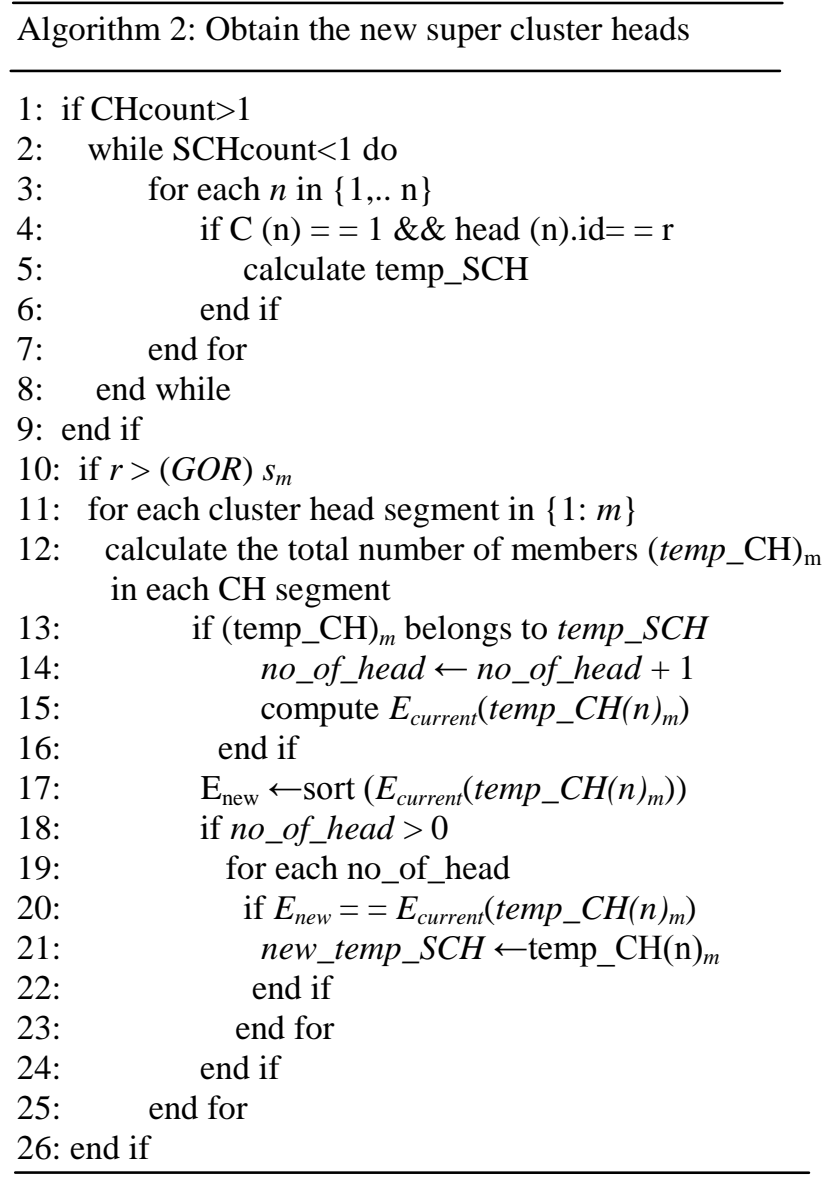

Once the heads are elected at both levels, the super $\mathrm{CHs}$ will advertise themselves by broadcasting $\mathrm{S}_{\mathrm{CH}}$ ADV_Msg over a distance $\sqrt{ } 2 \mathrm{M}$ using a nonpersistent CSMA protocol for association with lower level $\mathrm{CHs}$ as their member. This ADV broadcast message is of length $1_{\text {adv. }} \mathrm{CH}$ nodes then choose the nearest $\mathrm{S}_{\mathrm{CH}}$ and transmit Join_S $\mathrm{S}_{\mathrm{CH}}$ Msg to it again using CSMA through spreading code with spreading factor $h$. When $\mathrm{CHs}$ are associated, they will advertise themselves 
Through CH_ADV_Msg of length $1_{\text {adv }}$ for association with normal sensor nodes. A normal sensor node chooses a nearest $\mathrm{CH}$ node and sends a Join_CH_Msg to it. A CH must inform the super $\mathrm{CH}$ that how many members of normal sensor nodes have been associated with it, so that on the basis of this information, super $\mathrm{CH}$ can prepare the TDMA schedule for whole cluster. When the TDMA schedule is created, $\mathrm{S}_{\mathrm{CH}}$ node broadcasts it over a distance $\sqrt{ } 2 \mathrm{M}$ through TDMA_Schedule_Msg using spreading code. After TDMA schedule is distributed among whole cluster nodes, the steady-state phase runs. The data flow is then in the opposite direction starting from non- $\mathrm{CH}$ nodes (normal nodes) to the $\mathrm{CH}$ nodes using scheduling, aggregating and routing it to the super $\mathrm{CH}$ and thus it is finally the responsibility of super $\mathrm{CH}$ to forward sensor data received from lower level to the sink or base station. But in case of no $\mathrm{S}_{\mathrm{CH}}$ being selected, the $\mathrm{CHs}$ node will directly transmit the data to the BS.

\section{Simulations AND ANALYSIS}

\section{A. Preliminaries}

The following assumptions are made in this paper:

- The sink is located at some distance from the sensor field and its location is known by each node.

- All sensor nodes in the network are given equal amount of energy and their energies cannot be recharged.

- All sensor nodes communicate directly with the sink.

- Sensor nodes are location unaware.

- Symmetric propagation model exists.

- Cluster heads perform data aggregation.

- Sensor nodes that are randomly deployed in the region.

- All the nodes and the sink are stationary after deployment.

\section{B. Simulation environment}

The newly proposed protocol is simulated using Matlab simulator. At first, $\mathrm{N}=100$ number of nodes are deployed randomly in $M \times M$ square area with $M=200 \mathrm{~m}$. All nodes are given equal amount of initial energy for the following two cases under consideration i.e. Case1: $0.5 \mathrm{~J} /$ node and Case 2: $1 \mathrm{~J} /$ node. The base station is fixed at $(100,500)$. The sensor field is divided into five segments of same width according to the distance in $\mathrm{Y}$ direction from the base station. The radio model used is as described in the LEACH protocol. As in [5] and [2931], we use the values of $\delta=87.7 \mathrm{~m}$, length of data signal, $1_{\text {data }}=500$ bits, $\varepsilon_{\mathrm{fs}}=10 \mathrm{pJ} / \mathrm{bit} / \mathrm{m}^{2}, \varepsilon_{\mathrm{mp}}=0.0013 \mathrm{pJ} / \mathrm{bit} / \mathrm{m}^{4}$, $\mathrm{E}_{\mathrm{DA}}=5 \mathrm{~nJ} / \mathrm{bit} /$ signal, $\mathrm{E}_{\mathrm{mac}}=0.05 \mathrm{~J}, 1_{\mathrm{adv}}, 1_{\text {join }}, 1_{\mathrm{TDMA}}$, each of length of 200bits, and spreading factor, $\mathrm{h}=8$.

\section{Performance evaluation and analysis}

In this simulation study thirty runs of the simulator for each protocol are conducted and the average of these observations is plotted. The performance of the proposed protocol is measured at various times i.e. when the first node dies (FND), time when $25 \%, 50 \%, 75 \%$ nodes die out and time when $90 \%$ of the nodes are dead.

Fig. 1 shows the 100 sensors distribution in a field of size $200 \mathrm{~m} \times 200 \mathrm{~m}$. The network is divided into five segments of width $40 \mathrm{~m}$ each along $\mathrm{Y}$ direction with segment 1 being from (0-40) $\mathrm{m}$ and so on up to segment 5 being (160-200) m. Fig. 2 illustrates the probability of node $p_{i}$ which is obtained from equation (2). As can be seen, $p_{i}$ here varies inversely with distances. The sensor node which is at minimum distance to $\mathrm{BS}$ is having the maximum value of $p_{i}$, i.e. 0.0825 and the sensor node at the farthest distance from BS is having the minimum value of $p_{i}$, i.e. 0.0229 .



Fig.1. 100 Node Random Network

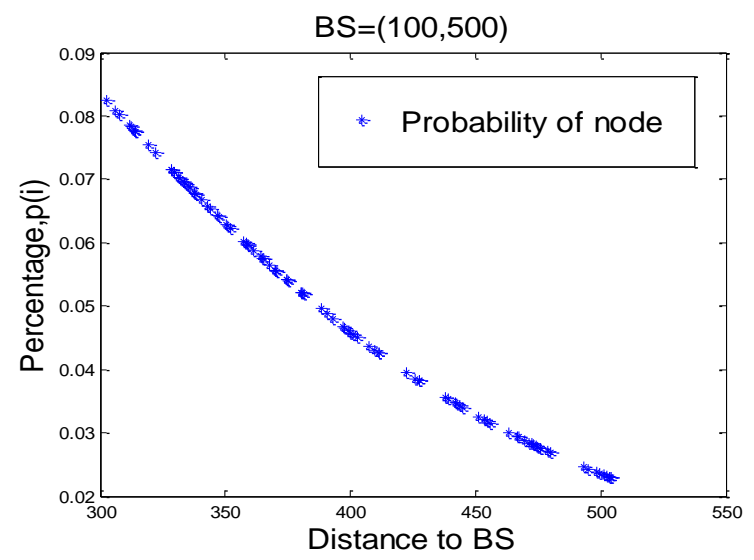

Fig.2. $p_{i}$ of Nodes

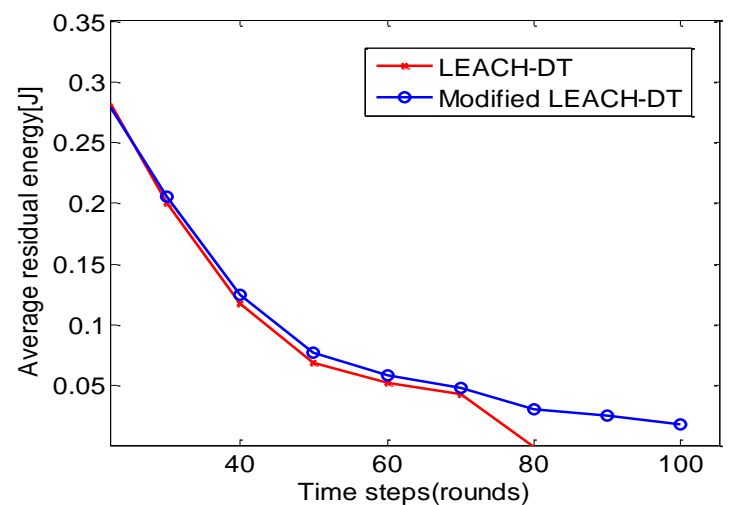

Fig.3. Residual Energy Analysis When Initial Energy / Node: 0.5J 


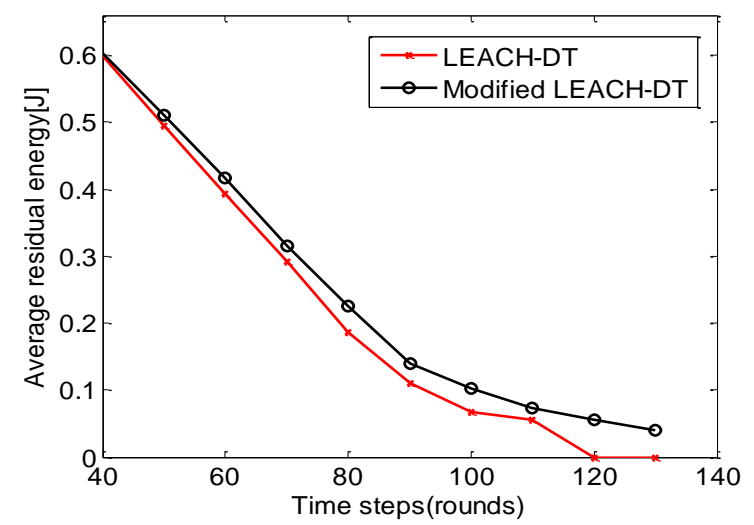

Fig.4. Residual Energy Analysis When Initial Energy / Node: 1J

Fig. 3 and 4 provides the amount of average residual energy in the network for LEACH-DT and proposed protocol with initial energy per node given as $0.5 \mathrm{~J}$ and1.0J respectively. From these figures it can be observed that modified LEACH-DT scheme has higher amount of residual energy as compared to LEACH-DT scheme. The energy saving in our proposed algorithm results due to selection of $\mathrm{CH}$ which is having higher residual energy and is likely to be closer to $\mathrm{BS}$ as compared to other $\mathrm{CH}$ selected in LEACH-DT.

Fig. 5 shows the minimum and maximum residual energy after communication round 60 in the various segments of the network for LEACH-DT and the proposed scheme when initial energy of $1 \mathrm{~J}$ was given to each node of the network. It is observed that difference between minimum and maximum of residual energy is less in all segments of the network in case of Modified LEACH-DT scheme as compared to that of LEACH-DT. This illustrates that our proposed protocol is balancing the communication load more efficiently than LEACHDT protocol. This energy balance has been achieved by our energy aware cluster head selection policy.

The results of the system lifetime and the network lifespan are shown in Fig. 6 and 7 for the LEACH-DT and the proposed protocol for the two cases. These results are a sample run out of several simulation runs.

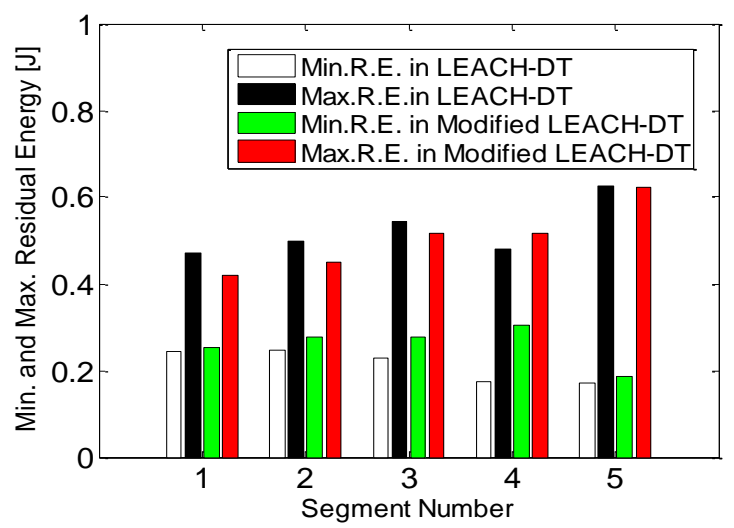

Fig.5. Minimum and Maximum Residual Energy

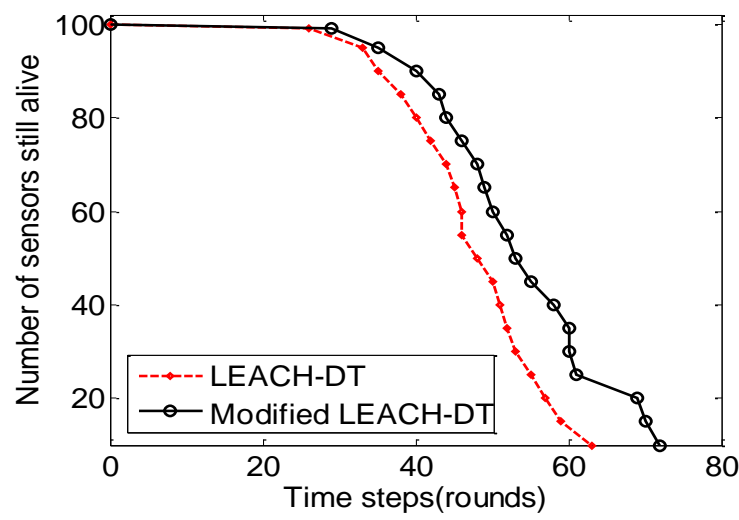

Fig.6. Network Lifetime When Initial Energy: 0.5J/Node

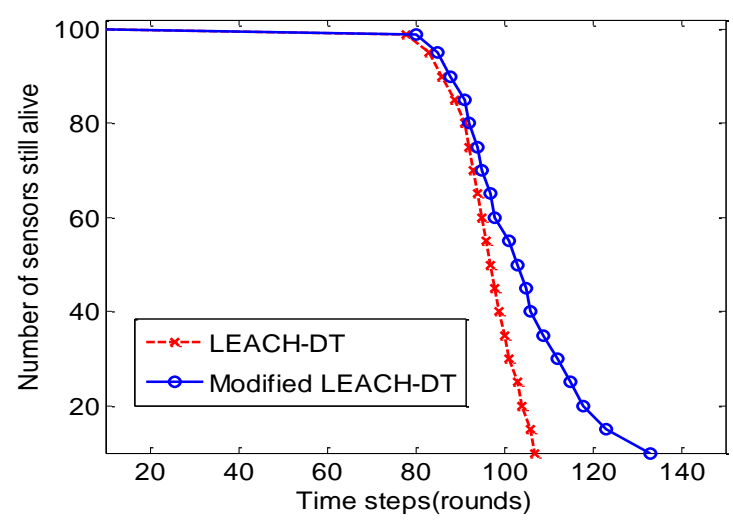

Fig.7. Network Lifetime When Initial Energy: 1J/Node

This balancing of communication load has increased the lifetime of the sensor network. As it is evident in Fig. 6 and 7, the nodes are alive for longer time in case of proposed protocol. Fig. 8 and 9 shows the performance comparison. Comparison is made between LEACH-DT and proposed scheme using metrics First Node Dead (FND), i.e. when $1 \%$ of the sensors in the network die out, time when $25 \%$ of the sensors die out, time when $50 \%$ of the sensors are alive (HNA), time when $75 \%$ sensors die and time when $90 \%$ of the sensors in the network are dead. In the first experiment, initial energy $0.5 \mathrm{~J} /$ node was given to each sensor and the network simulation was carried out to evaluate the performance of the proposed scheme. In the second experiment, initial energy $1.0 \mathrm{~J} /$ node was given in order to evaluate the performance shown in Fig. 9. As can be seen, FND for LEACH-DT and proposed scheme for the considered sensor network with BS at $(100,500) \mathrm{m}$ and initial energy $0.5 \mathrm{~J} /$ node is at 29 and 31 communication rounds, whereas for the second case with initial energy of $1.0 \mathrm{~J} /$ node, FND is at 78 and 85 and 78 rounds for LEACH-DT and modified LEACH-DT scheme respectively for the case 1 of initial energy of $0.5 \mathrm{~J} /$ node and 110 and 143 rounds respectively for the case 2 of $1.0 \mathrm{~J} /$ node.

Fig. 8 and 9 illustrates that proposed protocol, modified LEACH-DT increases the lifetime of the network by $8 \%$ for FND and by $29 \%$ for $90 \%$ nodes dead. 


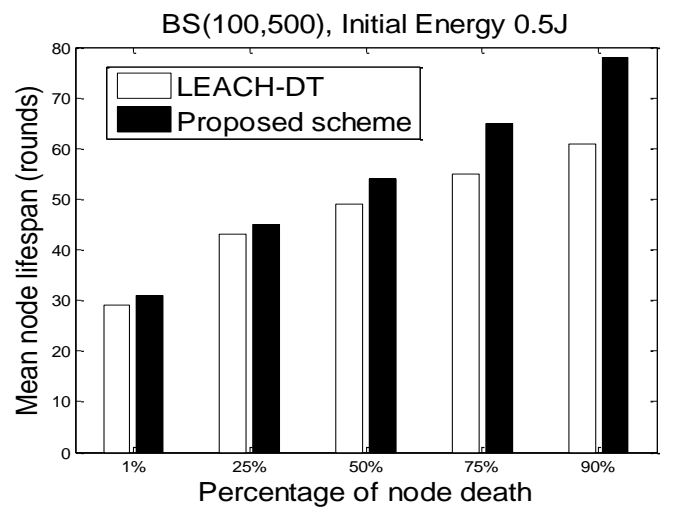

Fig.8. Performance Results When Initial Energy: .5J/Node

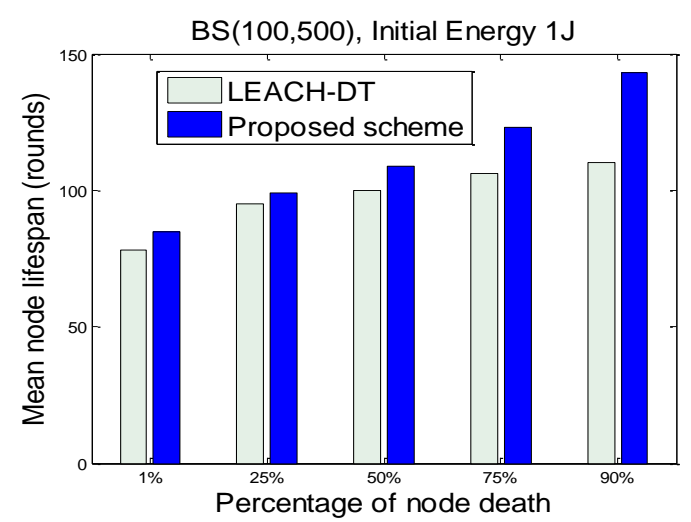

Fig.9. Performance Results When Initial Energy: 1J/Node

As discussed in section IV, two-level hierarchical extension of our proposed scheme was also developed and its performance was evaluated A sample run of the proposed hetero-hierarchical modified LEACH-DT scheme is shown in Fig. 10 and 11, for the two cases i.e. case 1: Initial energy $0.5 \mathrm{~J} /$ node and case $2: 1.0 \mathrm{~J} /$ node.

Fig. 12 and 13 shows the performance comparison for LEACH-DT and proposed Hetero-Hierarchical modified LEACH-DT scheme in terms of lifespan for the two cases i.e. with initial energy $0.5 \mathrm{~J} /$ node and $1.0 \mathrm{~J} /$ node respectively. After averaging the results of several trials, FND here comes out to be at 34 and 86 rounds for Hetero-Hierarchical modified LEACH-DT scheme for the case of $0.5 \mathrm{~J} /$ node and $1.0 \mathrm{~J} /$ node respectively. The results for $90 \%$ nodes dead (PND) are 99 rounds and 174 rounds for Hetero-Hierarchical modified LEACH-DT scheme for the case of $0.5 \mathrm{~J} /$ node and $1.0 \mathrm{~J} /$ node respectively.

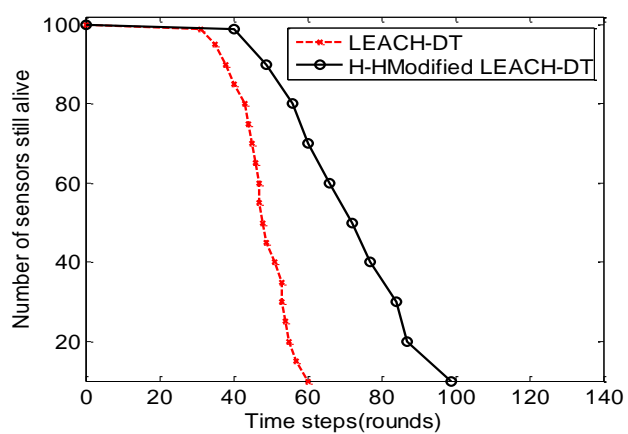

Fig.10. Network Lifetime When Initial Energy: 0.5J/Node

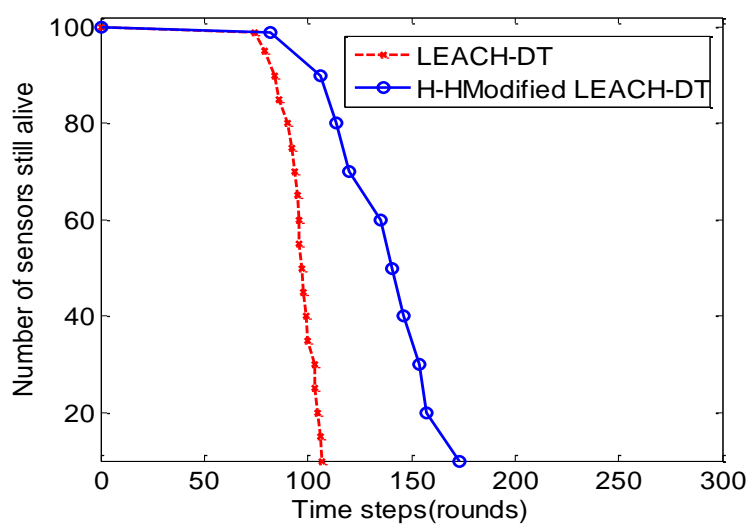

Fig.11. Network Lifetime When Initial Energy: 1.0J/Node

Simulation results indicate that there is increase in system lifetime in case of extending the proposed modified LEACH-DT protocol to two-level hierarchical architecture. Here the cluster heads among themselves formed another layer of clusters called super clusters before reaching to the base station.

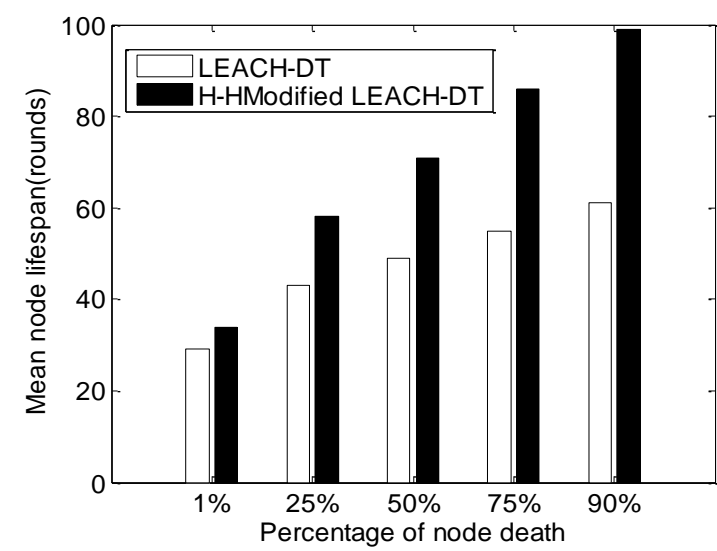

Fig.12. Performance Results When Initial Energy: 0.5J/Node

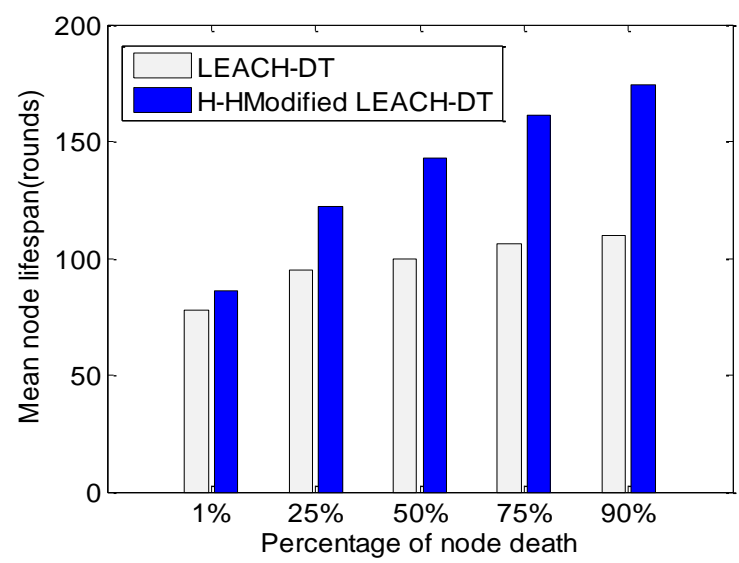

Fig.13. Performance Results When Initial Energy: 1J/Node

The proposed hetero-hierarchical modified LEACHDT scheme could achieve significant energy savings because now data is being forwarded through super cluster heads and not through direct transmission of cluster heads. Simulation results indicate that network 
lifespan with proposed hetero-hierarchical modified LEACH-DT scheme is about $13 \%$ better with respect to FND and about $60 \%$ better with respect to $90 \%$ nodes dead (PND) than with that of LEACH-DT protocol.

\section{CONCLUSIONS}

In this paper, a novel energy balanced and energy efficient topology management protocol for wireless sensor networks has been proposed. The proposed protocol is a modification of LEACH-DT protocol. The increase in system lifetime (FND) in the proposed protocol has been about $8 \%$ and about $29 \%$ for PND i.e. $90 \%$ node death. The proposed scheme outperforms LEACH-DT as it takes into account the remaining energy of each node during selection of final cluster heads, and is found to be more energy balanced approach. The hetero-hierarchical modified LEACH-DT scheme improves the network lifespan by about $13 \%$ in FND and about $60 \%$ in PND. It also improves the distance of $\mathrm{CH}$ communications with base station via a hierarchical approach and thereby provides scalability. It is expected that the proposed protocol may be used for sensors deployed for serving the assessment of the battlefield or disaster areas in future.

\section{REFERENCES}

[1] I F Akylidiz, W Su, Y Sankarasubramaniam, E Cayirci. Wireless sensor networks: a survey. Computer Networks 2002; 38 (4): 393-422, DOI: 10.1016/s13891286(01)00302-4.

[2] E Lule, T E Bulega. A scalable wireless sensor network (WSN) based architecture for fire disaster monitoring in the developing world. International Journal Computer Network and Information Security, 2015; 7 (2):40-49.

[3] W.B Heinzelman. Application-specific protocol architectures for wireless networks. 2000; Ph.D. dissertation, Massachusetts Institute of Technology, Cambridge.

[4] O Younis, M Krunz, S Ramasubramaniam. Node clustering in wireless sensor networks: Recent developments and deployment challenges. IEEE Network 2006; 20(3), 20-25, DOI: 10.1109/MNET.2006.1637928.

[5] W Heinzelman, A Chandrakasan, H Balakrishnan. Energy-efficient communication protocol for wireless microsensor networks. Proceedings of Hawaii International Conference on System Sciences 2000; 1-10, 10.1109/HICSS.2000.926982.

[6] W Heinzelman, a Chandrakasan, H Balakrishnan. Application-specific protocol architecture for wireless microsensor networks. IEEE Transactions on Wireless Communications 2002; 1(4): 660-670, DOI: 10.1109/TWC.2002.804190.

[7] M Tong, M Tang. LEACH-B: An improved LEACH protocol for wireless sensor network. Proceedings of International Conference on Wireless Communications Networking and Mobile Computing 2010; 1-4, DOI: 10.1109/WICOM.2010.5601113.

[8] W Xinhua, W Sheng. Performance comparison of LEACH and LEACH-C protocols by NS-2. Proceedings of International Symposium on Distributed Computing and Applications to Business Engineering and Science 2010; 254-258, DOI: 10.1109/DCABES.2010.58.
[9] S Fengjun. A Distributed Clustering Algorithm for wireless sensor networks. Wuhan University Journal of Natural Sciences 2008; 13(4), 385-390, DOI: 10.1007/s11859-008-0401-0

[10] J Hong, J Kook, S Lee, D Kwon, S Yi. T-LEACH: The method of threshold-based cluster head replacement for wireless sensor networks. Information Systems Frontiers 2009; 11(5), 513-521, DOI: 10.1007/s10796-008-9121-4.

[11] X Guo, L Chen. A variable round mechanism for routing protocols based on LEACH. Proceedings of International Conference on Wireless Communications, Networking and Mobile Computing 2008; 1-4, DOI: 10.1109/Wicom.2008.907.

[12] RK Tripathi, YN Singh, NK. Verma. N-LEACH, a balanced cost Cluster-Heads Selection Algorithm for Wireless Sensor Network. Proceedings of IEEE National Conference on Communications 2012; 1-5.

[13] A Azim, M.M Islam. Hybrid LEACH: A relay node based Low Energy Adaptive Clustering Hierarchy. Proceedings of IEEE Malaysia International Conference on Communications 2009; 911-916, DOI: 10.1109/MICC.2009.5431462.

[14] LQ Guo, Y Xie, CH Yang, ZW Jing. Improvement on LEACH by combining adaptive cluster head election and two hop transmission. Proceedings of International Conference on Machine Learning and Cybernitics, 2010; 1678-1683, DOI: 10.1109/ICML.2010.5580988.

[15] MB Yassein, A Al-zou'bi, Y Khamayseh, W Mardini. Improvement on LEACH Protocol of Wireless Sensor Network (VLEACH). Proceedings of International Journal of Digital Content Technology and its Applications 2009; 3, 132-136, 10.1109/SENSORCOMM.2007.4394931.

[16] M.H Awaad, WA Jebbar. Extending the WSN lifetime by dividing the network area into specific zones. International Journal of Computer Network and Information Security 2015; 7 (2):33-39.

[17] M Azharuddin, P Jana. A distributed algorithm for energy efficient and fault tolerant routing in wireless sensor networks. Wireless Networks, vol. 21, no.1, 2015, 251267, DOI: 10.1007/s11276-014-0782-2.

[18] MJ Handy, M Haase, D Timmerman. Low energy adaptive clustering hierarchy with deterministic cluster head selection. Proceedings of IEEE Conference on Mobile and wireless Communication Networks 2002; 368372, DOI: 10.1109/MWCN.2002.1045790.

[19] G Gupta, M Younis. Load-balanced clustering of wireless sensor networks. Proceedings of IEEE International Conference 2003; 3: 1848-1852, DOI: 10.1109/ICC.2003.1203919.

[20] O Younis, S Fahmy. HEED: A hybrid energy-efficient distributed clustering approach for Ad Hoc sensor networks. Trans. on Mobile Computing 2004; 3(4): 366379, DOI: 10.1109/TMC.2004.41.

[21] M Ye, C Li, G Chen, J Wu. EECS: An energy-efficient cluster scheme in wireless sensor networks. Proceedings of IEEE International workshop on Strategies for Energy efficiency in Ad Hoc and Sensor Networks, Phoenix, Arizona, USA 2005; 535-540, 10.1109/PCCC.2005.1460630.

[22] Y Wang, B Ramamurthy. Layered Clustering Communication Protocol for Wireless Sensor Networks. Proceedings of International Conference on Computer Communications and Networks 2007; 844-849, DOI: 10.1109/ICCCN.2007.4317923.

[23] D Kumar, TC Aseri, RB Patel. EEHC: Energy Efficient heterogeneous clustered scheme for wireless sensor 
networks. Computer Communications 2009; 32(4): 662667, DOI:10.1016/j.comcom.2008.11.025.

[24] Di Xin. A Novel Coverage-preserving Clustering algorithm for wireless sensor networks, Physics Procedia 2012; 33: 1054-1059, DOI: 10.1016/j.phpro.2012.05.173.

[25] P Kuila, PK Jana. Energy-Efficient Load-Balanced Clustering Algorithm for wireless sensor network. Proceedings of ICCS, Procedia Technology 2012; 6: 771777, DOI: 10.1016/j.protcy.2012.10.093.

[26] Z Xu, Y Yin, J Wang. A density based energy efficient clustering algorithm for wireless sensor networks. Proceedings of International Journal of Future Generation Communication and Networking 2013; 6: 75 85.

[27] NH Mak, WKG Seah. How long is the lifetime of a wireless sensor network? Proceedings of International Conference on Advanced Information Networking and Applications 2009; 763-770, DOI: 10.1109/AINA.2009.138.

[28] N Amini, M Fazeli, SG Miremadi, MT Manzuri. Distance-based segmentation: an energy-efficient clustering hierarchy for wireless micro-sensor networks. Proceedings of Annual Conference on Communication Networks and Services Research 2007; 18-25, DOI: 10.1109/CNSR.2007.27.

[29] S Kang, T Nguyen. Distance based thresholds for cluster head selection in wireless sensor networks. IEEE Communications Letters 2012; 16: 1396-1399, DOI: 10.1109/LCOMM.2012.073112.120450.

[30] V Gupta, R Pandey. Research on energy balance in hierarchical clustering protocol architecture for WSN. Proceedings of International Conference on Parallel, Distributed and Grid Computing 2014; 115-119. DOI: 10.1109/PDGC.2014.7030726.
[31] http.//www.ece.rochester.edu/ wheinzel/research.html\#co de.

\section{Authors' Profiles}

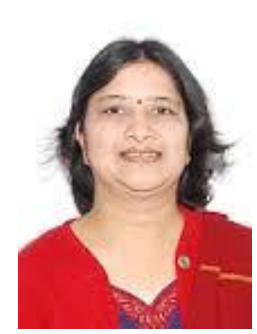

Vrinda Gupta received the B.E. and M.Tech. degree in Electronics and Communication Engineering in 1987, and 1994 from Nagpur University and Kurukshetra University (erstwhile R.E.C. Kurukshetra), India. She is currently pursuing her Ph.D. and is an Associate Professor in the Electronics and Communication Engineering Department at National Institute of Technology, Kurukshetra, India. Her research interests are in the fields of computer networking, and wireless sensor networks.

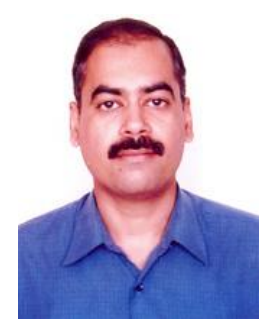

Rajoo Pandey received the B.E., M.Tech. and Ph.D. degrees in Electronics and Communication Engineering in 1989, 1991, and 2001 from Government Engineering College, Jabalpur, R.E.C. Kurukshetra, and Indian Institute of Technology, Roorkee, India, respectively. He is Professor and at present Head in the Department of Electronics and Communication Engineering, N.I.T. Kurukshetra, India. His research interests include signal and image processing and communication system.

How to cite this paper: Vrinda Gupta, Rajoo Pandey,"Modified LEACH-DT Algorithm with Hierarchical Extension for Wireless Sensor Networks", International Journal of Computer Network and Information Security(IJCNIS), Vol.8, No.2, pp.32-40, 2016.DOI: 10.5815/ijcnis.2016.02.04 